\title{
The Color of Complexes and UV-vis Spectroscopy as an Analytical Tool of Alfred Werner's Group at the University of Zurich
}

\author{
Thomas Fox and Heinz Berke*
}

\begin{abstract}
Two PhD theses (Alexander Gordienko, 1912; Johannes Angerstein, 1914) and a dissertation in partial fulfillment of a PhD thesis (H. S. French, Zurich, 1914) are reviewed that deal with hitherto unpublished UV-vis spectroscopy work of coordination compounds in the group of Alfred Werner. The method of measurement of UV-vis spectra at Alfred Werner's time is described in detail. Examples of spectra of complexes are given, which were partly interpreted in terms of structure (cis $\leftrightarrow$ trans configuration, counting number of bands for structural relationships, and shift of general spectral features by consecutive replacement of ligands). A more complete interpretation of spectra was hampered at Alfred Werner's time by the lack of a light absorption theory and a correct theory of electron excitation, and the lack of a ligand field theory for coordination compounds. The experimentally difficult data acquisitions and the difficult spectral interpretations might have been reasons why this method did not experience a breakthrough in Alfred Werner's group to play a more prominent role as an important analytical method. Nevertheless the application of UV-vis spectroscopy on coordination compounds was unique and novel, and witnesses Alfred Werner's great aptitude and keenness to always try and go beyond conventional practice.
\end{abstract}

Keywords: Coordination compounds · History of chemistry $\cdot$ Structure of complexes $\cdot$ Werner, Alfred · UV-vis spectroscopy

\section{The Color of Coordination Compounds and UV-vis Spectroscopy}

It is an intriguing fact that coordination compounds show great variation in colors, the greatest range of all classes of chemical substances. The colorful appearance of complexes is one of their prominent properties. Therefore it is not surprising that Alfred Werner, the founder of coordination chemistry, came across the spectral analysis ('Spektralanalyse') or as it is denoted today, UV-vis spectroscopy, which allowed colors of coordination compounds to be examined physically more precisely, particularly of those prepared in Alfred Werner's group.

Using color as an analytical indicator for a specific matter has a long history. Alchemists had already a certain idea about the analytical potential of colors in order to estimate substrate properties, since in those times colored elixirs have been said to be especially effective for

${ }^{\star}$ Correspondence: Prof. Dr. H. Berke Chemisches Institut, Universität Zürich Winterthurerstrasse 190, CH-8057 Zürich E-mail: hberke@chem.uzh.ch medical or ritual aims. In the 18 th century the fundaments of modern chemistry were laid and at the end of the 19th century the relationship between color and constitution of chemical compounds had been realized and systematically investigated with the aid of prism spectrometers. Indeed, from the early 1890 s on UV-vis spectroscopy became fully developed for chemical applications, particularly in the fields of organic chemistry and for solid inorganic materials. The scope and the analytical potential of UV-vis spectroscopy became fully visible after the turn of the century.

Therefore it is not surprising that Alfred Werner adopted this analytical method, using it for the characterization of 'his' complexes. From 1890 on, an increasing number of complexes was prepared in his group and almost daily new coordination compounds were added to the list, most of them had color. Alfred Werner was apparently aware of this possibility to analyze complexes by UV-vis spectroscopy, but despite this, he never turned it into a unique, routine type method to determine structures of 'his' complexes. Of course UV-vis spectroscopy was time consuming at Alfred Werner's time, needed great investments in apparatus and was expensive, since, as we will see later, it required an extensive silver photographic analysis and there were alternative methods that were easier to apply and to unravel the constitution ('Konstitution') of complexes. In addition, as we will see in the next section, the physical theory of light absorption at the time was ill-defined and could not easily be taken as a conceptual base for structural assignments of chemical compounds. This may be taken as the reasons why Alfred Werner only gave three students $\mathrm{PhD}$ thesis topics that dealt with UV-vis spectroscopy and the goal to develop this spectroscopic method for the structural assignments of complexes.

\section{Historical Context of UV-vis Spectroscopy at the Time of Alfred Werner}

The elementary set-up of the measuring equipment for UV-vis spectroscopy has not changed fundamentally up to the present day: A light beam passing through a medium sustains loss of intensity due to absorption effects of the media. Subsequent splitting into the spectral components via a prism allows comparison of the wavelength-dependent residual intensity with the original one and, subsequently conclusions on the nature of the absorbing media are drawn.

As already mentioned the seminal findings of Alfred Werner within the field of 
coordination chemistry were predestined to quite some extent for spectroscopic investigations. However, at the time the concept of the physical mechanisms of light absorption was fairly vague and had its foundation mainly in the atomic model of Rutherford, whereby negatively charged electrons orbited around positively charged nuclei. In the global view of those former times, light propagated like an acoustic wave through a mysterious vacuum medium, the so-called 'ether', suffering deceleration and absorption while striking an electron. Alexander Gordienko, a coworker of Alfred Werner's group, wrote in his $\mathrm{PhD}$ thesis (1912): "Considering the interior reason for light absorption of resolved matter, the opinions are diverging here, as well. According to Baly the light absorption is caused by periodical motions of the electrons between two connected atoms. He calls this phenomenon 'isorhopesis', which is closely related to the oscillation of the double bonds in benzene. Another hypothesis, which is nowadays acknowledged to be most likely, stems from Stark. According to him, there are positively charged spheres on the surface of an atom and above mobile negative electrons, which are arranged at certain locations. He distinguishes saturated, non-saturated and loose electrons. - We are interested first and foremost in the loose electrons, because the saturated and non-saturated ones show, due to theoretical calculation, just an absorption in ultra red and inaccessible ultra violet, respectively. Stark supposes the loose electrons to be the centers of selective absorption; each of the loose electrons corresponds to a certain band in the absorption spectrum." - At the time the atom model of Bohr was as yet not established, whereby electrons occupy certain atomic orbits of discrete energies, and where transitions between the orbits lead to absorption or emission of appropriate light with distinct frequencies. - This model was still unknown and even more the principles of quantum mechanics, which were not developed until the 1920s. In spite of the lack of a valid theoretical background for Werner's spectroscopic investigations his revolutionary findings in coordination chemistry appear all the more remarkable.

\section{Early Theories of the Origin of Color}

At the turn of the 19th century, lots of empirical rules were established in order to classify the influence of substituents, solvents, molecular constitution, tautomerism or salification on the color of compounds. As pioneers in this area Gordienko cites in his $\mathrm{PhD}$ thesis scientists like Armstrong, Graebe, Hantzsch, Kaufmann, Kehrmann, von Konstanecki, Liebermann, Nietzki,
Thiele and Witt. Their rules served also the other PhD students of the Werner group, who dealt with UV-vis spectroscopy, as a base for 'Investigations about the Relationships between Color and Constitution of Chemical Compounds' (PhD thesis, A. Gordienko[1]), for investigations 'About Absorption Spectra of Metal Ammonias' (PhD thesis, J. Angerstein ${ }^{[2]}$ ), or research on 'The Absorption Spectra of certain Chromium Salts' (in partial fulfillment of a $\mathrm{PhD}$ thesis, H. S. French $\left.{ }^{[3]}\right)$. It should be noted at this point that these $\mathrm{PhD}$ theses are unique in the sense that they were never published by Alfred Werner in scientific journals.

At the time of Alfred Werner it was already known that "the presence of certain groups determines the absorption potential of a given compound." Witt named such groups chromophores, a denotation and definition that is valid up to now. Chromophores convert colorless substances into colored compounds, so-called chromogenes. If a compound contains additional auxochromic groups, its color experiences further intensification. Gordienko emphasized "the most important groups", like $=C-N O,=C=S,-N=N-,-N_{2} O-,-N O$, - $C O$ - or $-C=C$-, and described their influences in a purely phenomenological manner. Concerning the CO-group, he noted for instance: "Principally, this group is too weak in order to generate any color. There have to be present at least two of such groups, namely positioned close to each other". From a modern point of view, such a description is of course unsatisfactory, because it is based on subjective observations without explanation based on a physical background. However, for an early absorption spectroscopist, such a notation was certainly a helpful qualitative guidance in order to distinguish between compounds containing no, one or even two carbonyl groups.

According to Gordienko, compared to auxochromic groups “...there have been found also some of that ilk, which brighten the corporal color after introduction into a chromogene. Therefore, we have to distinguish between two kinds of groups: 1 . Bathochromes. They deepen the color of chromogens (...), according to the following scheme: greenish-yellow, yellow, orange, red, purple, brown, violet, indigo blue, cyan blue, bluish green, green. 2. Hypsochromic groups. They brighten up the color or raise it. As a consequence of their entry we get the opposite sequence of colors: green, blue, red, ..., yellow." In other words: This observation of color changes during chemical reactions was envisaged to serve as an analytical indicator in order to evaluate the nature of reactants and products and their interconversions.

The effects of auxochromic groups were explained by Kaufmann simply “... in such a manner, that they influence the unsaturated state of the chromogene... ." Gordienko himself gave an example by means of the influence of some substituents onto the absorption properties of benzene, "... which is no rigid, dead and unchangeable ballast, but an extremely sensitive entity." In addition to auxochrome groups, the knowledge about the relationship between the constitution of compounds and their color was of analytical relevance, as well. In his PhD thesis, Gordienko quoted e.g. ortho-benzochinone, which is red in contrast to its yellow para isomer.

Some other empirical rules explained the influence of salification on the auxochromic character of amino and hydroxide groups (Kaufmann), as well as the changes, which undergo auxochromes while participating in tautomeric processes (rearrangement theory by Hantzsch) in the presence of different alkaline and alkaline earth metals. Of particular interest with respect to Werner's investigations on the dissociation behavior of metal complexes was, however, the Hantzsch concept of side valences. Accordingly, auxochromic groups deploy their color deepening capacity by aid of side valences, leading to bond formation to neighboring chromophores. This is in fact an important aspect of Werner's chemistry: A great number of his complexes contained amino groups, attached by nitrogen side valencies - nitrogen lone electron pairs - to the central metal atom. The strong influence of amino groups on complex colors was certainly most welcome to Werner's group, due to their sensitivity enhancing effect in spectroscopic measurements.

At Werner's time such measurements were based mainly on visual and quite subjective and therefore qualitative methods and were often carried out with hand spectroscopes and other insensitive instruments, until a completely new, quantitative method came up, which had been developed by W. N. Hartley. The Hartley spectrometer served the Werner group for plenty of exceedingly fruitful absorption measurements and was extensively used by Gordienko, who gave a detailed description about principals and instrumentation in his PhD thesis. The other two PhD students were then dealing with UV-vis spectroscopy in a more applied way, unraveling structures of complexes by spectroscopic comparison.

\section{The Hartley Spectrometer: Principles and Instrumental Setup}

Alexander Gordienko arrived in Alfred Werner's group apparently well trained in physics, since he had studied at the 
'Imperial College of Railway Engineers' in St. Petersburg. He turned himself into a specialist in absorption spectroscopy and he laid the foundation to UV-vis spectroscopy in Werner's group, probably by contacting Alfred Werner's former supervisor at the ETH Zurich, Professor Hantzsch, who meanwhile had moved to the University of Leipzig. Hantzsch studied UV-vis spectroscopy of organic compounds from 1907 onwards. The experimental setup as described in Gordienko's $\mathrm{PhD}$ thesis, as well as his general remarks concerning the principles of data evaluation, were brought into Alfred Werner's group by him and were also cited by the other coworkers of Alfred Werner working in the spectroscopic field. One part of Gordienko's PhD thesis reads as follows: “... For this purpose I used a Zeiss spectrograph: A spectroscope, which allows to photograph the spectra. The apparatus was set up for ultra violet light; I used a quartz prism, quartz lenses and quartz cuvettes. As a source of light served an electric iron arc, which has been generated by aid of two iron rods of 1 cm thickness, under usage of 40 volts and ca. 8 Amp. In order to inflame the lamp, I allowed the iron rods to touch each other for a moment and increased then the distance between them. At first I recorded the spectrum of the iron arc itself onto a photographical plate; then I positioned the cuvettes (path thickness 20, 10, 5, 2, $1 \mathrm{~mm}$ ) with the solution between iron arc lamp and slot (=2) and photographed now the iron arc light after passing the liquid. The ready plate has been evaluated under the microscope, and I determined the $a b$ sorption limits in $\mathrm{nm}$ (...). The illumination amounted all about 3 sec."

The above-described photographical method according to Hartley was sheer revolutionary for those times, because it afforded finally insight into the ultra-violet (UV) range of light, which is invisible to the human eye. Spectroscopic measurements were, therefore, no longer limited to applications under use of visible light.

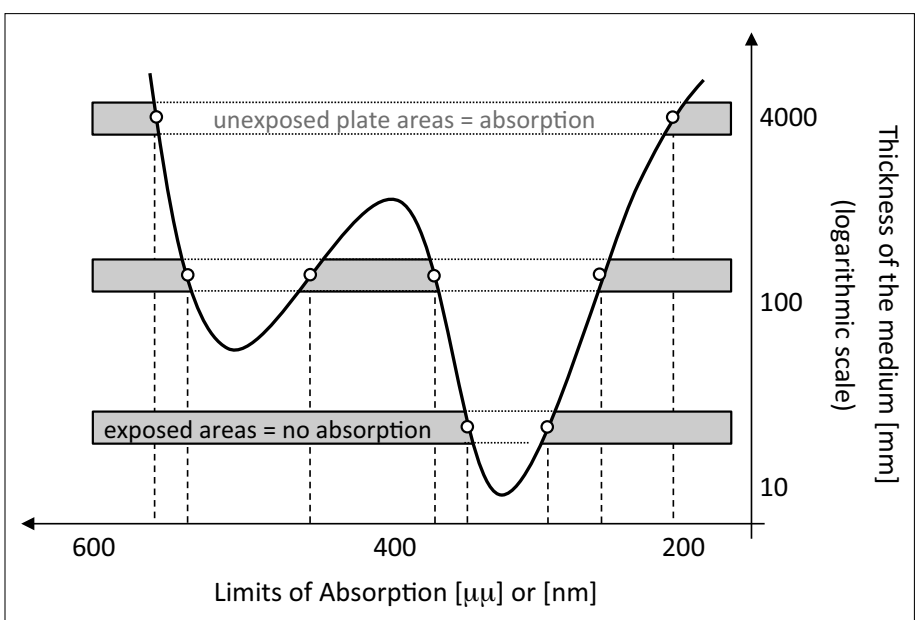
modern instruments.
In order to display the UV absorption behavior of a liquid medium as a function of the light wavelength, its spectrum was obtained on the basis of a series of samples with different coat thicknesses. Blackened areas on the photographical disk implied aperture of appropriate wavelengths, whereas unaffected areas indicated light absorption. The latter areas broadened with increasing thickness of the samples and subsequent graphical display of the absorption limits (in $\mathrm{nm}$ ) against the coat thickness (in mm) resulted in Hartley molecular vibration curves, which were strikingly close to spectra taken nowadays with

Alexander Gordienko described the basic principles of the instrumental setup in his thesis as follows (Fig. 1): "One photographs e.g. the spectrum of the iron arc, putting the cuvettes containing $1 / 1000$ $n$ solutions in between (coat thicknesses: $1,2,5,10,20,50 \mathrm{~mm}$ ). Afterwards one takes a more concentrated solution, e.g. $1 / 10 n$ and photographs again for coat thicknesses of 1,2, 5, 10,20,50 mm. As long as Beer's law is valid - and this is the case, if the solvent is very permeable and the solute durable - one can claim: An absorption spectrum of a $1 / 1000$ n solution has been recorded under constant illumination for coat thicknesses of 1, 2, 5, 10, $20,50,100,200,500,1000,2000,5000$ $\mathrm{mm}$. One puts the logarithmic values of the coat thicknesses onto the ordinate axis and the limits of absorption onto the axis of abscissae. A connection of the resulting points gives the so-called molecular vibration curve."

\section{Typical UV-vis Studies Described in PhD Theses of Alfred Werner's Group}

The repeatedly cited $\mathrm{PhD}$ thesis of Gordienko from 1912 is quite worthy to be highlighted in this context, not only because it is the fruitful result of sheer untiring efforts in the field of preparative chemistry and analytical spectroscopy, but also because it is a pioneer work containing at the time valid cornerstones for the assignment of UV-vis absorption spectra.

Gordienko addressed e.g. additions of nitrogen-containing compounds to carbon-carbon double bonds in order to gain insight into the nature of the resulting bonds within the generated "nitro corpora”. Werner considered three possible alternatives:

i) Polynitro corpora and aromatic hydrocarbons are possibly connected via aromatically bound hydrogen.

ii) The benzene ring of the polynitro corpora is involved.

iii) The $\mathrm{NO}_{2}$-group is involved.

After more than 400 (!) meticulously journalized color reactions like "2.4.6-trinitro-toluene + benzyl-naphthylamine: red needles" Gordienko's final result was, that "the occurrence of a color in combination with the recently discussed regularities leads to the conclusion, that these colored compounds (which are just durable in solution) possess the same constitution for aromatic and aliphatic nitro corpora, that they are generated under saturation of the side valences between the nitro group and the unsaturated carbon atoms (gap bonding) and that they have, as already mentioned, to be expressed in the following manner:"

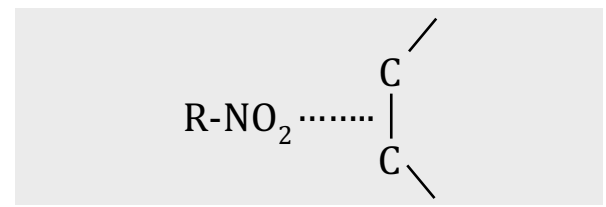

Gordienko deduced this result in a purely combinatory manner, bringing the observed colors and intensities into a systematical relation with the number of nitro groups and double bonds of the formed adducts.

However, similar experiments of solutions of hydrocarbons in tetranitromethane under application of Hartley's spectroscopic method failed:

"Due to the very strong absorption of Fig. 1. Schematic plot of a UV-vis spectrum on the basis of photographical plates. tetranitromethane itself, Beer's law is not valid in this case and, therefore, we are unable to obtain a continuous Hartley curve (...). As far as we can see, these spectroscopic measurements didn't yield a lot."

In another test series, consisting of 65 separate experiments, Gordienko investigated bathochromic, hypsochromic and sterical effects of some functional groups such as $-\mathrm{OH},-\mathrm{OCH}_{3},-\mathrm{CH}_{3},-\mathrm{HCO}$, $-\mathrm{COOH}$ and $-\mathrm{COOR}$. The conclusion was, that "The introduction of $-\mathrm{OH},-\mathrm{OCH}_{3}$ and - $\mathrm{CH}_{3}$ groups deepens an already existing color of a compound. -OH is the strongest 
bathochromic group, followed by $-\mathrm{OCH}_{3}$ and finally $\mathrm{CH}_{3}(\ldots$.$) . -\mathrm{HCO},-\mathrm{COOH}$ and -COOR have hypsochromic effects."

A further series of 44 separate experiments was carried out, in order to classify the reaction mixtures with respect to their color effect: "We returned to the idea of bringing together the described nitro corpora with phenols and other aromatic substrates, either directly (eventually under melting), or in acetone solution. The following coloration nuances had to be distinguished: a) almost insensible yellow, b) very weak lime yellow, c) weak lime yellow, d) lime yellow, e) cadmium yellow, f) chromium yellow, g) orange."

At this point the general dilemma of any analytical chemist emerges when working without spectroscopic support and, instead, basing his observations solely on subjective impressions. In the actual case Gordienko had to distinguish between almost imperceptible color differences - a rather doubtful undertaking, considering individually different and subjective visual perception. How to distinguish seven colors in an objective and reliable manner, if six of them are yellow nuances...?

For that reason the chapter 'About the Relation between Constitution and Color of Cobaltiaces' of Gordienko's thesis is by far the most important with respect to absorption spectroscopy, since Gordienko describes the UV absorption curves of a huge number of cobalt complexes. These have been recorded with the aid of a Hartley spectrometer in order to classify ligand influences on the absorption behavior of an appropriate entire complex. Altogether, 34 cobalt complexes have been measured, yielding an appropriately large number of ligand effects, which have been described very explicitly. The effect of substitution of ' $\mathrm{CO}_{3}$ ' for 'en' or ' $\mathrm{NH}_{3}$ ' is for example described in Fig. 2.

It should be mentioned that Alfred Werner and his coworkers had already been able to differentiate cis and trans isomers. In the spectrum in Fig. 3 Gordienko distinguishes between the cis (....) and trans $(-)$ form of $\mathrm{Co}(\mathrm{en})_{2}(\mathrm{Cl})_{2}$, where the former has an absorption maximum at $530 \mathrm{~nm}$ and the latter at 470 and $615 \mathrm{~nm}$.

On the basis of the spectra of a few known cis and trans isomers they were able to determine the structure of unknown compounds by simple comparison of the appropriate band shapes. Such efficient work gave surely an additional thrust on the way to Nobel Prize in $1913 . .$.

Another example for the impressive analytical potential of absorption spectroscopy in the Werner group may be given by means of a series of spectra, which were recorded by Helen French. She investigated a multiplicity of chromium salts and recorded also absorption spectra of chro-

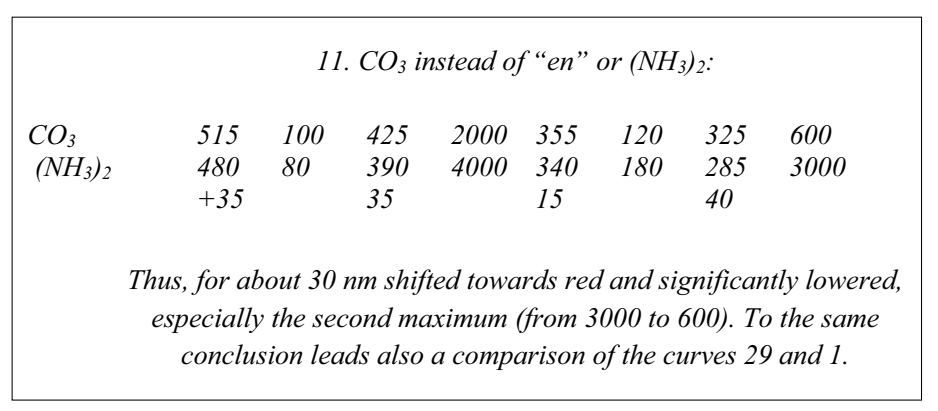

Fig. 2. Excerpt from

$\mathrm{PhD}$ thesis of $\mathrm{A}$. Gordienko.

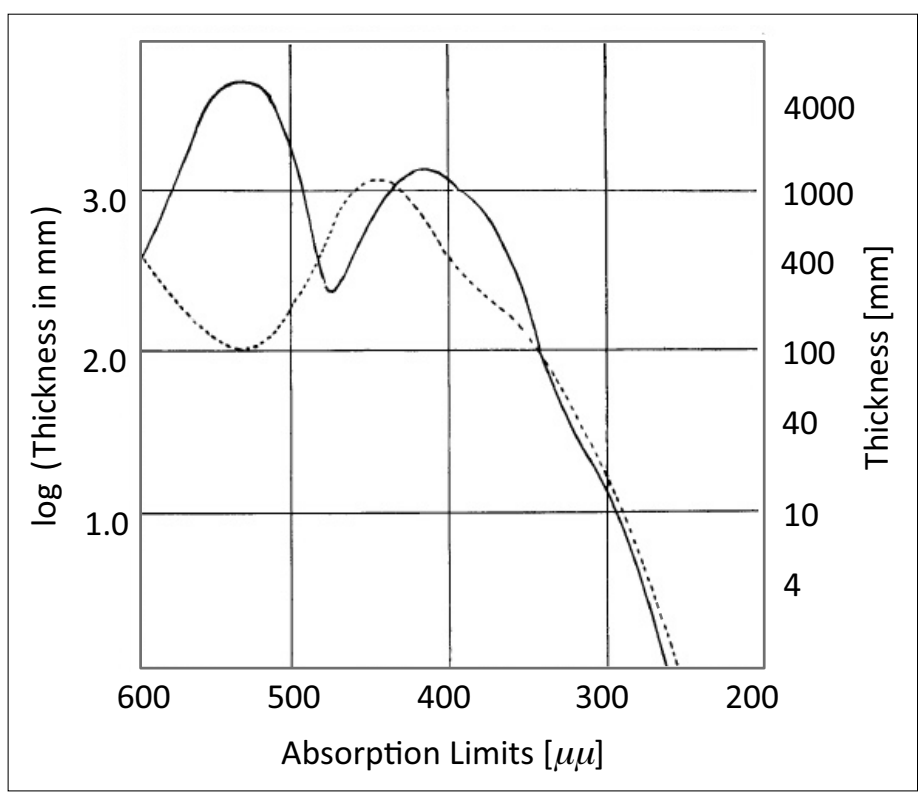

Fig. 3. UV-vis spectra of the cis (-...) and trans (-) forms of $\mathrm{Co}(\mathrm{en})_{2} \mathrm{Cl}_{2}$ from the $\mathrm{PhD}$ thesis of $\mathrm{A}$. Gordienko.

mium hydrates with a different number of ammonia ligands. In doing so, she observed an increasing displacement of the absorption curve toward ultra-violet by the successive substitution of $\mathrm{NH}_{3}$-groups for water ligands (Fig. 4).

Fig. 4 shows a series of spectra that are typical for octahedral structures. Helen French observed a large band separation between $\left(\mathrm{H}_{2} \mathrm{O}\right)_{6}$ and $\left(\mathrm{NH}_{3}\right)_{6}$ complexes, as well as absorption bands of mixed complexes between these limits (absorption maxima $A$ and $\mathrm{C}$ ). She comments this as follows: "The general nature of the $a b$ sorption is the same in all six curves, in that each contains two well-defined $a b$ sorption bands. As the number of $\mathrm{NH}_{3}-$ groups increases, and the number of $\mathrm{OH}_{2}$ groups correspondingly decreases, the absorption bands are moved steadily toward the ultra-violet, showing that the $\mathrm{OH}_{2}$-group, containing oxygen, deepens the color (bathochrome), while the $\mathrm{NH}_{3}$ group, containing nitrogen, has the opposite effect (hypsochrome), and moves the absorption bands toward the shorter wave lengths. Moreover, these two qualitatively opposed effects are quantitatively nearly equal."

This is just a lean description of the observations, because at that time there was no knowledge of the relation between nature of ligands and absorption behavior of the appropriate complex: In the actual case a substitution of ammonia for water leads to increasing field strength of the ligand sphere. This results in an extension of HOMO-LUMO separations, causing higher frequent electron transfers and, therefore, the observed blueshifts.

Also J. Angerstein, the third coworker of Werner who dealt with UV-vis spectroscopy, merely expressed some amazement and left it at a short description instead of risking a misinterpretation, when comparing the spectra of three different cobalt complexes (Fig. 5): "It's quite peculiar, that the first minima in the visible range are absolutely not influenced by the substituents, whereas the pentammonium salts are pretty sensitive. The first maxima at $425 \mathrm{~nm}$ and $430 \mathrm{~nm}$ also don't show any particular difference; only in the ultra-violet range substitution gains more importance, where the chloroaquatetrammonium salt exhibits selective absorption, whereas the bromoaquatetrammonium salt absorbs just continuously. Therefore, the introduced water molecules have a stabilizing effect onto the absorption in the visible spectral range."

Looking with the eyes of a UV-vis spectroscopist today at Fig. 5, the mentioned "selective absorption" of the chloro complex in the ultraviolet range $(340 \mathrm{~nm})$ leads to a band shape that is remarkably 
close to typical three-band spectra of undistorted octahedral structures like the also presented tris(trimethylenediamine) cobalt complex [CoTr${ }_{3}$. But during those early times UV-vis-spectroscopy was still in its infancy and Angerstein was not yet able to draw the obvious conclusion, that the chloro complex might have a less distorted octahedral structure than the bromo complex.

\section{Conclusion}

Similar to the developments of conductivity measurements and the casting of a coordination chemistry theory, the application of UV-vis spectroscopy in Alfred Werner's group witnesses Werner's great aptitude and keenness to always try and go beyond conventional practice. Although he fostered the use of routine-type methodologies in his group, he apparently felt obliged to tread uncharted and unexplored paths regardless of any foreseeable difficulties. Unfortunately at Werner's time, physical chemistry lacked reliable background knowledge in many respects, particularly in this case the mechanisms of light absorption by matter so that an indepth interpretation of UV-vis spectra was deemed impossible. Consequently, the scientific value of UV-vis spectroscopy was limited in his group to data acquisition and spectra descriptions, which might have been one reason why this method did not play such a prominent role for Werner and his coworkers. He even did not publish this work. But still the spectra could have been used to identify complexes in solution. However, for this purpose the method was too laborious and time-consuming even at Werner's time, because other methods would have served the same purpose with much less expenditure. Nevertheless, the enormous data output of UV-vis spectra of French, Gordienko and Angerstein, combined with their scientific ingenuity, gave presumably also support to Werner's revolutionary three-dimensional view of metal complexes.

Received: March 10, 2014

[1] A. Gordienko, 'Untersuchungen über die Beziehungen zwischen Farbe und Konstitution chemischer Verbindungen', Inaugural-Dissertation, Zürich, Gebr. Lehmann \& Co., 1912.

[2] J. Angerstein, 'Ueber die Absorptionsspektren von Metallammoniaken', Inaugural-Dissertation, Zürich, J. J. Meier, 1914.

[3] H. S. French, 'The Absorption Spectra of Certain Chromium Salts', in partial fulfillment of a $\mathrm{PhD}$ thesis, Zürich, 1914.

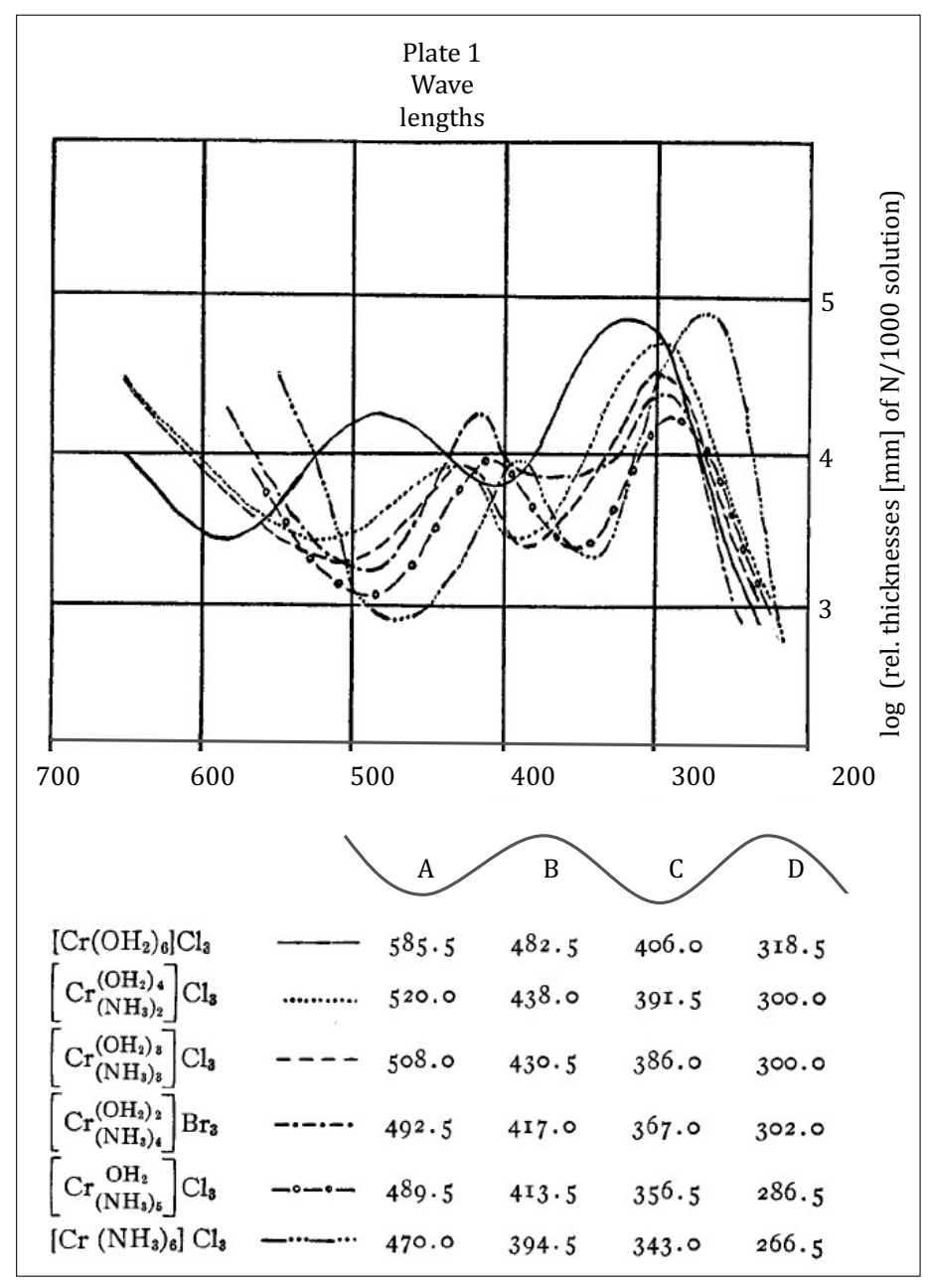

Fig. 4. Absorption spectra of chromium halide hydrates with a different number of ammonia ligands. $H$. French, in partial fulfillment of $\mathrm{a} P \mathrm{PD}$ thesis.

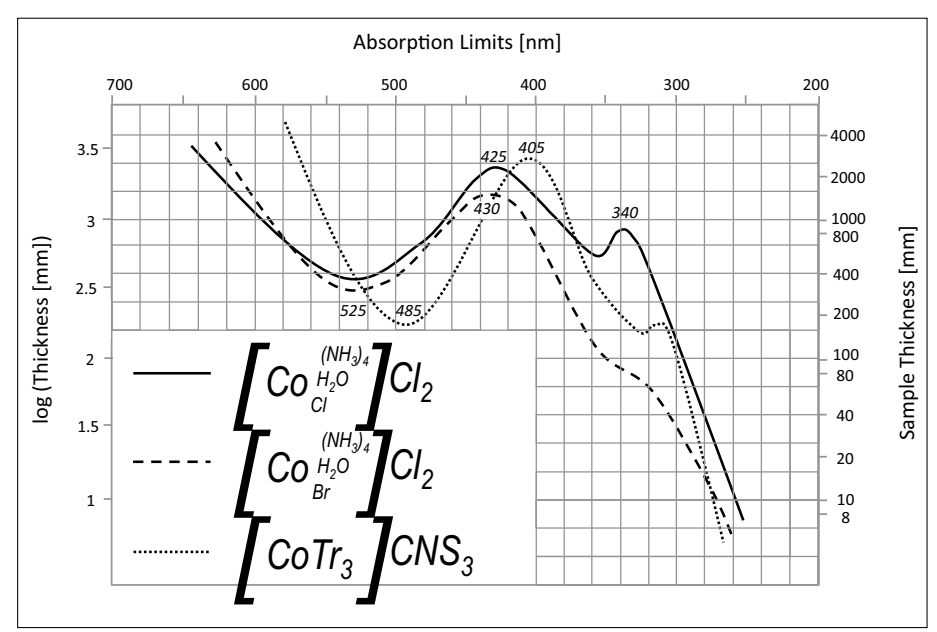

Fig. 5. Three UV-vis spectra of different cobalt complexes from the $\mathrm{PhD}$ thesis of $\mathrm{J}$. Angerstein. 